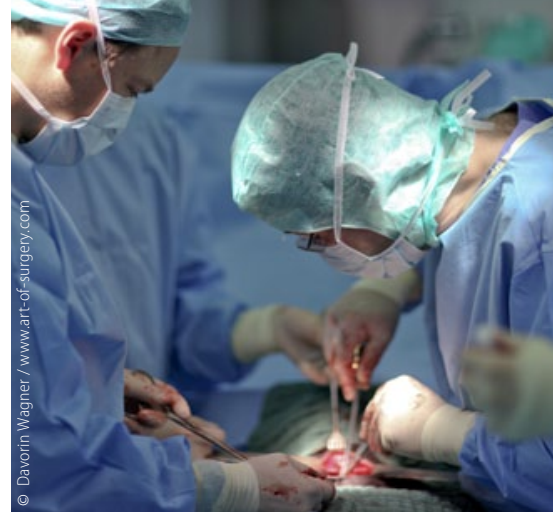

in einer randomisierten multizentrischen Studie, ob laparoskopische Eingriffe bei Rektumkarzinomen vergleichbar gute Ergebnisse liefern wie offene Operationen. Dazu operierten sie im Rahmen der Phase-III-Studie COLOR II (Colorectal cancer Laparoscopic or Open Resection II Trial) 739 Patienten mit Rektumkarzinom minimalinvasiv und 364 klassisch offen. Eingeschlossen wurden Patienten ohne Hinweise auf Fernmetastasen mit einem Tumor, der sich weniger als $15 \mathrm{~cm}$ vom Anus entfernt befand. Die Daten zum primären Endpunkt, die Rezidivrate nach drei Jahren, liegen noch nicht vor. Aber die Studienautoren um Martijn van der Pas, Amsterdam, Niederlande, konnten schon die Daten zu sekundären Endpunkten auskopisch kompletter Resektionen: Sie war in der laparoskopischen Gruppe nicht signifikant schlechter als in der Gruppe mit offener Operation (88 vs. $92 \%$ ). Die Rate mikroskopisch erfolgreicher Resektionen - kein Nachweis von Tumorzellen in den äußeren $2 \mathrm{~mm}$ des exzidierten Gewebes lag in beiden Gruppen bei $90 \%$. Wie zu erwarten, war der Blutverlust bei der minimalinvasiven Operation geringer (median 200 vs. 400 ml), die Darmfunktion kam schneller wieder in Gang (2 vs. 3 Tage) und die Patienten konnten schneller nach Hause (nach 8 vs. 9 Tagen). Die Sterberate war nach 28 Tagen in der Gruppe mit minimalinvasiver Operation niedriger (1 versus $2 \%$ ), aber nicht signifikant. Die Rate postoperativer Komplikationen unter-
Offen oder laparoskopisch operieren? Diese Frage stellt sich auch bei Rektumkarzinomen.

schied sich ebenfalls nicht signifikant (40 vs. $37 \%$ ). Die Studienautoren sehen die laparoskopische im Vergleich zur offenen Operation bezüglich Sicherheit und Resektionsergebnis als nicht unterlegen an. Jedoch scheinen sich die Patienten nach dem minimalinvasiven Eingriff deutlich schneller zu erholen.

Thomas Müller

van der Pas MHGM et al. Laparoscopic versus open surgery for rectal cancer (COLOR II): shortterm outcomes of a randomised, phase 3 trial. Lancet Oncol. 2013;14(3):210-8.

\section{kurz notiert}

Immuntherapie +++

„In jedem Menschen gibt es sogenannte

WT1-reaktive T-Zellen, die bestimmte Leukämieformen bekämpfen können“, erklärt Matthias Wölfl, Würzburg. „Für die therapeutische Wirkung ist es offenbar ganz entscheidend, auf welche Weise man die T-Zellen im Labor aktiviert", so Wölfl. Kommen die T-Zellen bei ihrer Aktivierung mit Interleukin-21 in Kontakt, sind sie später langlebiger und damit auch länger gegen Tumorzellen aktiv. Im Blut der Patienten waren sie bis zu ein Jahr lang nachweisbar. Fehlte dagegen bei der Aktivierung das Interleukin, waren sie schon nach weniger als vier Wochen verschwunden (Aude G et al. Science Translational Medicine. 2013, DOI: 10.1126/scitransImed.3004916).

\section{mTOR-Protein +++}

Das Protein «mammalian Target of Rapamycin» (mTOR) kontrolliert Prozesse wie Zellwachstum und Stoffwechsel. Als Hauptbestandteil von mTORC1 und mTORC2 stimuliert es die Produktion von Proteinen und Fetten. Fehlregulationen des mTOR-Signalnetzwerks sind u. a. an der Entstehung von Krebs beteiligt. So könnte die Suche nach bislang unbekannten mTOR-Proteinen neue Ansatzpunkte zur Behandlung von Krebs aufzeigen. Aaron Robitaille und Kollegen [Robitaille AM. Science.2013, DOI: 10.1126/science.1228771] konnten nun mehr als 300 neue mTOR-Zielproteine identifizieren, die ein breites Spektrum von Aufgaben erfüllen.

Fall-Kontroll-Studie

\title{
Tabakrauch macht Blasenkrebs aggressiv
}

Rauchen ist nicht nur ein Risikofaktor für Blasenkrebs. Laut einer Studie haben Raucher bei Diagnose auch bösartigere Tumoren als Nicht- oder Exraucher. Die Untersuchung war Teil des englischen „West Midlands Bladder Cancer Prognosis Programme“, in das Patienten mit abnormen zystoskopischen Befunden einbezogen werden. Ausgewertet wurden die Daten von knapp 1.100 Patienten, 840 davon Männer. In $80 \%$ der Fälle lagen Urothelkarzinome vor. Raucher $(\mathrm{n}=227)$ waren im Schnitt bei der Blasenkrebsdiagnose vier Jahre jünger als lebenslange Nichtraucher ( $\mathrm{n}=224 ; 65,7$ vs. 69,7 Jahre). Ehemalige Raucher $(n=616)$ waren im Durchschnitt 72,8 Jahre alt. Im Mittel waren die Tumoren von Rauchern $3,31 \mathrm{~cm}$, jene von Nichtrauchern 2,84 cm groß. Das Tumorstadium bei Diagnose lag rechnerisch bei 1,81 für Raucher, 1,77 für Exraucher und 1,56 für Nichtraucher. Auch die Zahl der Tumoren unterschied sich mit 2,08 (Raucher) und 2,10 (Nichtraucher) nicht signifikant. Exraucher waren bei Diagnose älter als Nichtraucher. Auch Zigarren- und Pfeifenraucher waren zum Zeitpunkt der Diagnose älter als Studienteilnehmer, die nie Tabakprodukte konsumiert hatten. Erklären können die Forscher diese Ergebnisse nicht, sie empfehlen aber eine vorsichtige Interpretation.

Laut den Wissenschaftlern machen es die neuen Erkenntnisse unter anderem erforderlich, Blasentumoren von Rauchern besonders gründlich zu resezieren - unter Einschluss von Detrusormuskulatur, um etwaige Muskelinvasivität $\mathrm{zu}$ erfassen. Außerdem sei es wahrscheinlich, dass der Verzicht aufs Rauchen nach der Blasenkrebsdiagnose die Prognose von Patienten verbessere. Auf Harnblasenmalignome entfallen in der Bundesrepublik 4,6\% aller Krebsneuerkrankungen von Männern (Platz 4) und 2,0\% aller neu diagnostizierten bösartigen Tumoren von Frauen (Platz 13). Das geht aus der letztjährigen Erhebung „Krebs in Deutschland“ hervor, vorgelegt vom Robert Koch-Institut und der Gesellschaft der epidemiologischen Krebsregister in Deutschland. Robert Bublak

van Roekel EH et al. Smoking is associated with lower age, higher grade, higher stage, and larger size of malignant bladder tumors at diagnosis. Int J Cancer. 2013 Jan 7. [Epub ahead of print] 